sighted proposals must not be squandered through professional inertia, short term financial expediency, or lack of managerial imagination.

Consultant in Community Child Health,

D M B HALL

St George's Hospital and Medical School

London SW 17 0QT

Director of Children's Services,

West Essex Health Authority,

Princess Alexandra Hospital,

Harlow CM20 IDT

1 Robertson J. Young children in hospital. London: Tavistock, 1958

2 Gilbert J, Robinson T, Littlewood JM. Home intravenous antibiotic treatment in cystic fibrosis. Arch Dis Child 1988;63:512-7.

3 Anderson HR. Trends and district variations in the hospital care of childhood asthma; results of a regional study 1970-85. Thorax 1990;45:431-7.

4 Hall DMB. Health for all children. Oxford: Oxford University Press, 1989.

5 Department of Health and Social Security. Fit for the future. London: HMSO, 1976. (Court report.)

\section{Cultured composite skin grafts for burns}

\section{Banked allografts offer a prospect of immediate wound coverage}

Each year more than 10000 people need admission to hospital in England and Wales for burns. ${ }^{1}$ Immediate excision and grafting in patients with extensive burns is thought to improve survival - a hypothesis supported by retrospective studies of both children ${ }^{2}$ and adults, ${ }^{3}$ although not confirmed by a prospective study. ${ }^{4}$ Early grafting would be expected also to reduce pain and the morbidity associated with sepsis, fluid loss, and scarring.

What methods of grafting are available? The commonest technique for resurfacing full thickness skin defects is with split thickness autografts, which may be expanded fourfold by meshing ${ }^{5}$ or 20 -fold by dicing. ${ }^{6}$ Unfortunately, patients with extensive burns may have few donor sites, but meshed skin expanded sixfold may be used by overlaying it with allograft ${ }^{7}$ (which if used alone does not survive in the long term, even if the recipient has been immunosuppressed ${ }^{8}$ or the antigenicity of the donor skin reduced ${ }^{9}$.

Fifteen years ago the problem of lack of graftable skin seemed close to solution with the description of a reliable technique for culturing large numbers of keratinocytes in vitro. ${ }^{10}$ The first reports of the use of these cultured autologous keratinocytes were encouraging, ${ }^{11}$ with expansions of up to 10000 -fold. ${ }^{12}$ The most obvious disadvantage was the three week interval between taking the donor skin sample and getting large sheets of cultured keratinocytes for grafting. There is some evidence that cultured keratinocytes have attenuated antigenicity, ${ }^{13}$ suggesting that cultured allogeneic keratinocytes might be used. These cells survive for less than a week, however, and may be acting as no more than elaborate dressings-albeit also secreting growth factors. ${ }^{14}$ Keratinocyte grafts have been found to have several persistent problems: blistering and contracture due to lack of dermis ${ }^{15}$ and an abnormal ultrastructure of the dermo-epidermal junction within seven months of grafting. ${ }^{16}$ These have limited their clinical application and provided further stimulus to the production of skin substitutes with a dermal component.

Cadaver skin allografts have given excellent results. ${ }^{17} 18$ When these are used the dermis survives and the epidermis may later be replaced with cultured autologous keratinocytes.
Unfortunately the demand for allograft skin outstrips supply in the United States by a factor of five to seven, ${ }^{19}$ and there is a real risk of transmission of infection. In an effort to overcome these problems a composite artificial skin substitute has been developed, consisting of a dermal component of bovine collagen and shark chondroitin-6-sulphate overlaid with a sheet of Silastic, ${ }^{20}$ which acts as a temporary epidermal layer but must later be replaced by a thin split skin autograft or cultured keratinocyte sheets. ${ }^{21}$ Use of this composite reduces fluid loss and contracture, and follow up at one year has shown results equivalent to those from split thickness autografts with respect to hypertrophic scarring and patient satisfaction, although graft take was less satisfactory. ${ }^{22} \mathrm{~A}$ variation of the technique uses cultured fibroblasts incorporated into the collagen-glycosaminoglycan membrane to emulate a normal dermis more closely. ${ }^{23}$ The Silastic dermal composite has the disadvantage of requiring two procedures to achieve epithelial cover.

The latest stage in this evolutionary process is the use of composite cultured skin grafts consisting of cultured keratinocytes on a collagen-fibroblast dermal equivalent. These have been evaluated in animals ${ }^{24}$ and, more recently, in patients with burns - but poor results were obtained owing to graft lysis. ${ }^{25}$ Further studies were more promising, ${ }^{26}$ although the use of autologous keratinocytes still resulted in a delay of a week and an expansion of only 20 -fold. Allogeneic fibroblasts and keratinocytes should overcome these problems and were evaluated in patients undergoing full thickness excision of tattoos. ${ }^{27}$ The grafts were not acutely rejected, and although the allograft cells may gradually be replaced by those of the host ${ }^{28}$ this should not pose a problem clinically unless there is ulceration and loss of the graft. After eight months the appearance of the composite grafts applied to burns was superior to that obtained with meshed autologous split thickness skin, and neither contractures nor hypertrophic scarring occurred. ${ }^{26}$

Several problems still need to be solved if cultured composite skin grafts are to be used widely. Many groups have reported poor handling characteristics and dermo-epidermal separation. A basement membrane develops in vitro only after prolonged culture, ${ }^{29}$ although hemidesmosomes form within six days if human type IV collagen is included in the dermal equivalent. ${ }^{30}$ Moreover, the take of the graft may be very variable in patients with burns. ${ }^{25}{ }^{26}$ Another important problem is the lack of an ideal dressing for the tenuously adherent epidermis. Clearly, the technique requires further development, but with improvements in the structure of the dermal component and inducing formation of the basement membrane in vitro the stage is set for the production of a true skin equivalent. The incorporation of skin adnexae remains an elusive dream, but the aim of providing banked skin allograft in unlimited quantities for the severely burnt patient should be attainable.

JAGDEEP NANCHAHAL Senior House Officer

DAI DAVIES

Consultant Plastic Surgeon

Department of Plastic and Reconstructive Surgery,

Charing Cross Hospital,

London W6 8RF

1 Muir IFK, Barclay TLK, Settle JAD. Burns and their management. 3rd ed. London: Butterworths, 1987.

2 Herndon DN, Parks DH. Comparison of serial debridement and autografting and early massive excision with cadaver skin overlay in the treatment of large burns in children. $\mathcal{f}$ Trauma 1986;26:149-51.

3 Tompkins RG, Hilton JF, Burke JF, et al. Increased survival after massive thermal injuries in adults: preliminary report using artificial skin. Crit Care Med 1989;17:734-40.

4 Thompson P, Herndon DN, Abston S, Rutan T. Effect of early excision on patients with major thermal injury. I Trauma 1987;27:205-7.

5 Tanner JC, Vandeput J, Olley JF. The mesh skin graft. Plast Reconstr Surg 1964;34:287-92.

6 Nanchahal J. Stretching skin to the limit: a novel technique for split skin graft expansion. Br f Plast Surg 1989;42:88-91. 
7 Alexander WJ, MacMillan BG, Law E, Kittur DS. Treatment of severe burns with widely meshed skin autograft and meshed skin allograft overlay. $\mathcal{F}$ Trauma 1981;21:433-8.

8 Frame JD, Sanders R, Goodacre TEE, Morgan BDG. The fate of meshed allograft skin in burned patients using cyclosporin immunosuppression. Br f Plast Surg 1989;42:27-34.

9 Alsbjörn BF, Sørensen B. Grafting with epidermal Langerhans' cell depressed cadaver split skin Burns 1985;11:259-63.

10 Rheinwald JG, Green H. Serial cultivation of strains of human epidermal keratinocytes: the formation of keratinizing colonies from single cells. Cell 1975;6:331-44.

11 O'Connor NE, Mulliken JB, Banks-Schlegel S, Kehinde O, Green H. Grafting of burns with cultured epithelium prepared from autologous epidermal cells. Lancet 1981 ; i:75-8.

12 Gallico GG, O'Connor NE, Compton CC. Permanent coverage of large burn wounds with autologous cultured human epithelium. $N$ Engl f Med 1984;311:448-51.

13 Morhenn VB, Benike CJ, Cox AJ, Charron DJ, Engleman EG. Cultured human epidermal cells do not synthesize HLA-DR. $f$ Invest Dermatol 1982;78:32-7.

14 Burt AM, Pallett CD, Sloane JP, et al. Survival of cultured allografts in patients with burns assessed with probe specific for $Y$ chromosome. BMf 1989;298:915-7.

15 Auböck J. Hautersatz durch Kultivierte Keratinozyten. Z Hautkr 1988:63:565-7.

16 Wubock J. Hautersatz durch Kulterte KR autografts show abnormal reconstitution of anchoring fibrils. FAMA 1988;259:2566-71.

17 Cuono C, Langdon R, McGuire J. Use of cultured epidermal autografts and dermal allografts as skin replacement after burn injury. Lancet 1986;i:1123-4.

8 Langdon RC, Cuono CB, Birchall N, et al. Reconstitution of structure and cell function in human skin grafts derived from cryopreserved allograft dermis and autologous cultured keratinocytes. f Invest Dermatol 1988;91:478-85.

19 May SR, Still JM, Atkinson RN. Recent developments in skin banking and the clinical uses of cryopreserved skin. I Med Assoc Ga 1984;257:233-6.

20 Burke JF, Yannas IV, Quinby WC, Bondoc CC, Jung WK. Successful use of a physiologically acceptable artificial skin in the treatment of extensive burn injury. Ann Surg 1981;194:413-28.

21 Boyce ST, Hansbrough JF. Biologic attachment, growth and differentiation of cultured human epidermal keratinocytes on a graftable collagen and chondroitin-6-sulphate substrate. Surgery 1988;103:421-31.

22 Heimbach D, Luterman A, Burke J, et al. Artificial dermis for major burns. A multi-cente randomized trial. Ann Surg 1988;208:313-20.

23 Hansbrough JF, Boyce ST, Cooper ML, Foreman TJ. Burn wound closure with cultured autologous keratinocytes and fibroblasts attached to a collagen-glycosaminoglycan substrate. fAMA 1989;262:2125-30.

24 Bell E, Sher S, Hull B, et al. The reconstitution of living skin. $\mathcal{F}$ Invest Dermatol 1983;81(suppl): 2-10.

25 Wassermann D, Schlotterer M, Toulon A, et al. Preliminary clinical studies of a biological skin equivalent in burned patients. Burns 1988;14:326-30

6 Hull BE, Finley RK, Miller SF. Coverage of full-thickness burns with bilayered skin equivalents: a preliminary clinical trial. Surgery 1990;107:496-502.

27 Nanchahal J, Otto WR, Dover R, Dhital SK. Cultured composite skin grafts: biological cultured skin equivalents permitting massive expansion. Lancet 1989;ii:191-3.

28 Gielen V, Faure M, Mauduit G, Thivolet J. Progressive replacement of human cultured epithelium allograft by recipient cells as evidence by HLA class I expression. Dermatologica 1987;175: 166-70.

29 Chamson A, Germain N, Claudy A, Perier C, Frey J. Study of basement membrane formation in dermal-epidermal recombinants in vitro. Arch Dermatol Res 1989;281:267-72.

30 Tinois E, Tiollier J, Dumas H, Thivolet J. In vitro and post-transplantation differentiation of human keratinocytes grown on human type IV collagen film of a bilayered dermal substitute [Abstract]. F Invest Dermatol (in press).

\section{Does passive smoking cause heart disease?}

\section{The evidence is strong enough to warrant measures to reduce exposure}

Active smoking is the most readily preventable cause of coronary heart disease. In 1986 the United States Surgeon General identified passive smoking as a cause of lung cancer and called for further research on the relation between passive smoking and cardiovascular disease. ${ }^{1}$ Since then six cohort studies $^{2-9}$ and one case-control study ${ }^{10}$ published in English have examined this association and an answer is beginning to emerge. The cohort studies were of variable size and included participants from the United States, ${ }^{2-57}$ Scotland, ${ }^{6}$ and Japan. ${ }^{89}$ Measurement of exposure to passive smoking was based on questionnaire responses; in only one study was there objective evidence of exposure to passive smoke and the same study had information on workplace exposure. ${ }^{+}$All but one study ${ }^{5}$ followed up individuals; and one study measured the stability of smoking rates part way through the follow up. ${ }^{7}$ Several coronary heart disease end points were examined, and all the studies tried to control for other risk factors.

Collectively the cohort studies suggest a positive association between passive smoking and death from heart disease with relative risks ranging from $1 \cdot 2$ to $2 \cdot 7$. There are several possible explanations for the observed association; these include chance, bias (including publication bias), and confounding - or the association could be causal. Chance is an unlikely explanation given the precision of the results in some studies; the combined relative risks were significant in both men and women."

Systematic error (bias) in measuring passive smoking is a possible explanation. ${ }^{12}$ If the passive smoking group included active smokers who had been incorrectly classified as nonsmokers the relative risk in this group would have been inflated. Only a small part of the increase in the risk of lung cancer associated with passive smoking among non-smokers, however, could have been due to this type of misclassification. ${ }^{13}$ Active smoking increases the risk of lung cancer by about 10-fold, but its effect on heart disease is much less (roughly a doubling of risk), so misclassification is highly unlikely to be the sole cause of the observed increase in the risk of heart disease associated with passive smoking. Furthermore, since many non-smokers who do not live with smokers are known to be exposed to smoke from other sources, particularly at work, the effect of passive smoking is likely to be underestimated..$^{13}$ Publication bias, the greater likelihood of studies with positive results to be published compared with those with negative results, does not explain the association of passive smoking with lung cancer ${ }^{14}{ }^{15}$; there is no reason to believe that it explains the association with coronary heart disease.

Confounding - that is, mixing of effects - is the most likely non-causal explanation for the observed association. Confounding might account for some or all of the association if passive smoking were associated in the population studied with other risk factors for heart disease and if these associations had been inadequately controlled in either the study design or the analysis. All six studies controlled for age, and four comprehensively controlled for the major cardiovascular disease risk factors ${ }^{2467}$; the impact on the relative risk of controlling for these risk factors was in general minimal. Since non-smokers tend to come from healthy families, however, the effects of unknown confounders might still be important. ${ }^{16} 17$

A judgment is required to determine whether the association is causal. The temporal association is correct; the association is plausible given our knowledge of the effects of active smoking and the effect of passive smoking on other health outcomes'; physiological and biochemical studies suggest possible mechanisms ${ }^{18}$; there is some evidence of a dose response relation ${ }^{67}$; and there is consistency of results among the cohort studies. Differences between sidestream and mainstream smoke, the absence of a truly non-exposed control group in studies of active smoking, and a greater susceptibility of passive smokers to the health damaging effects of tobacco smoke may explain the apparently high relative risks of coronary heart disease associated with passive smoking compared with the relative risks caused by active smoking.

The available evidence does therefore suggest that passive smoking is a cause of coronary heart disease. Nevertheless, further epidemiological studies are required in various settings. In particular there is a need for large, well designed case-control studies that accurately measure recent and past passive smoking at home and at work and adjust for all known potential confounders, particularly socioeconomic factors. Follow up studies of people at high risk, such as survivors of a myocardial infarction, may also be worth while.

From the public health perspective this association is important because coronary heart disease is much more common than respiratory disease; most of the deaths attributed to passive smoking in the United States and New Zealand have been caused by coronary heart disease. ${ }^{119}$ 